\title{
Dossier « Réaffectations du foncier : régulation étatique, investissements privés et initiatives citoyennes »
}

\section{Une terre commune dans le village? Relations village-État et économie morale au travers de la biographie d'une parcelle dans le centre du Myanmar}

\author{
Stéphen Huard
}

Anthropologie et Histoire, Université de l'East Anglia, Département de Développement international, Norwich Research Park, Norwich (NR4 7UG), Royaume-Uni

Mots-clés :

terre commune ; gouvernance foncière ; économie morale ; agriculture ; Myanmar/Birmanie

\begin{abstract}
Résumé - Reconstruire la biographie d'une terre dans un village du centre du Myanmar permet $\mathrm{d}$ 'analyser les rapports entre les relations foncières locales et les pratiques gouvernementales. Cette étude de cas se focalise sur une parcelle en particulier : détenue par un premier couple de villageois dans les années cinquante, puis confisquée et réattribuée par le gouvernement à un second couple, elle fut par la suite transformée en terre «vacante » et est aujourd'hui un terrain de football. Cependant, cette parcelle est dépendante d'usages locaux, lesquels ont été récemment confrontés à des enjeux de développement qui ont actualisé la question de son statut: terre commune, terre villageoise, ou terre vacante ? La biographie de cette parcelle montre d'une part comment l'enchâssement des usages et des statuts est lié à des pratiques gouvernementales qui sont confrontées aux relations foncières et à l'économie morale villageoises, et d'autre part comment le statut de cette terre est actuellement négocié.
\end{abstract}

Keywords: common land; land governance; moral economy; agriculture; Myanmar/Burma

\begin{abstract}
A common land in the village? Land biography, village-state interplay and moral economy in central Myanmar, Burma. Through the description of a biography of a land in a village located in central Myanmar, this paper analyzes the interplay between local land relations and government practices. In Myanmar, colonial and postcolonial land reforms and policies have partly changed land access for local populations. This case study focuses on a specific plot of land: originally held by a family of villagers in the 1950s, the plot was latter allocated by government agents to the couple who cultivated it via a land-lease arrangement. The land was afterward turned into a "vacant land" following the implementation of a military-socialist policy and is now the village's football ground. Meanwhile, the parcel remained dependent upon local uses and regulations challenged by development issues that update the question of its status: common land, village land, or vacant land? First, the plot's biography enables to show successive and overlapping uses and status. Second, the unfolding of government practices (colonialists, military-socialists and post-democracy) are analyzed and confronted to local land relations and moral economy. Finally, the role of village headman is highlighted in regard to how land status are claimed and negotiated, concluding on the importance of local-government relations to understand the dynamics of social changes at play in this area.
\end{abstract}


Suivre les changements d'usage et de statut d'une terre, suite aux politiques et réformes agraires mises en œuvre par l'État dans le centre du Myanmar, permet de comprendre les dynamiques en jeu entre l'État et les villages ${ }^{1}$. En effet, l'histoire des villages et les relations foncières qui s'y déploient sont liées aux pratiques des gouvernements successifs depuis la colonisation britannique et au-delà. Dans le village de Pawn Gyo ${ }^{2}$, le statut d'une terre - le terrain de football - est confronté depuis 2013 à un enjeu de développement local : l'acheminement de l'électricité au village pourrait être financé par la vente du terrain de football. Cependant, l'histoire de cette parcelle est complexe et nécessite de clarifier la succession et la superposition d'usages et de statuts dont elle (a) fait l'objet.

La trajectoire historique et sociale de cette terre est un exemple intéressant pour mettre en avant le contexte actuel des relations foncières dans la zone sèche du Myanmar. Pour ce faire, le statut actuel de la parcelle est questionné : terre publique, terre commune ou terre villageoise ? Interroger les catégorisations privé/public, commun/collectif amène à s'intéresser à l'usage du sol, c'est-à-dire aux modalités d'accès à cet espace, à la distribution et transmission des droits sur celui-ci. Mais cela incite également à se pencher sur les conditions de reproduction socioéconomique des unités sociales, sur les autorités juridico-politiques et enfin sur les groupes qui participent à la régulation foncière (Le Meur, 2002). Dans le village de Pawn Gyo, les relations foncières recouvrent un spectre allant de la régulation locale des transferts de droits sur la terre aux modes de reconnaissance de la propriété, en passant par la définition du statut des terres. Et de fait, l'histoire de cette parcelle à Pawn Gyo est inséparable des relations sociales qui s'y déploient.

Ces relations sont ancrées dans des temporalités spécifiques, dans une religiosité bouddhiste ainsi que dans des conceptions du juste liées aux conditions de vie, aux conditions agroécologiques et aux rapports de dépendance entre villageois. Le concept d'économie morale permet d'englober l'ensemble de ces éléments. À la suite des travaux pionniers d'Edward Palmer Thompson (1971) et de James Scott (1977), Didier Fassin définit l'économie morale comme « la production, la répartition, la circulation et l'utilisation des sentiments moraux, des émotions et des valeurs, des normes et des obligations dans l'espace

\footnotetext{
1 Cet article a été réalisé grâce à un terrain de recherche au sein de l'étude « Understanding rural land issues to engage comprehensive policy dialogue in Myanmar » organisée par le Groupe de recherche et d'échanges technologiques et financé par le Livelihoods and Food Security Trust Fund. Une version antérieure de cette recherche a été présentée lors de la septième édition des Journées des doctorants portant sur « les usages du sol » organisée par le LPED les 26 et 27 mars 2015 à Marseille.

2 Tous les noms de lieux ont été rendus anonymes.
}

social » (2009, p. 1257). On utilisera donc ce concept pour rendre compte de l'ancrage des usages dont a fait l'objet la parcelle en question par les villageois.

Au Myanmar, la période coloniale ${ }^{3}$ a été marquée par un effort de l'administration britannique pour accroître la production agricole et contrôler hommes et territoires via la transformation des dispositifs de régulation foncière (Huard, 2014 ; Thant Myint-U, 2001). Après l'invasion japonaise (1942-45) et le retour de l'administration anglaise (1945-48), le Myanmar est devenu indépendant avec à sa tête un gouvernement d'union nationale qui a entrepris la nationalisation de l'économie et des terres dans une lutte contre le landlordism ${ }^{4}$. Cette lutte s'est accrue avec le coup d'État militaire de 1962 à travers une politique de restitution des terres aux petits paysans et de suppression des intermédiaires privés entre l'État et les agriculteurs jusqu'à la fin des années quatre-vingt. Parmi les dispositifs de ce gouvernement militaro-socialiste, il faut signaler les multiples " comités " pensés comme des organes déconcentrés du gouvernement à l'échelon villageois (Taylor, 2009), le chef de village, la socialisation du secteur agricole à travers un réseau de coopératives étatiques et le système du procurement qui imposait des quotas sur les récoltes à partir du milieu des années soixante $^{5}$ (Warr, 2000 ; Steinberg, 1981). Les années quatre-vingt-dix sont marquées par la libéralisation du marché agricole et la privatisation graduelle de l'économie (Kurosaki, 2004). Depuis quinze ans environ, le délaissement relatif de la planification agraire fait écho au tournant vers un capitalisme militaro-financier (Woods, 2011, 2013). Cette tendance se répercute dans l'affaiblissement des structures gouvernementales de régulation (arrêt de prélèvement de taxes, arrêt du procurement, disparition des coopératives, faible suivi des cadastrages, etc.), mais aussi dans l'accroissement de la «petite corruption » des agents gouvernementaux par leur capacité à délivrer ou à créer certains services ${ }^{6}$. Après l'ouverture du pays et l'appel à une transition démocratique en 2011, 1'usage et le statut des ressources sont revenus sur le devant de la scène. Deux lois foncières

3 Période qui s'étend de 1812 à 1948 pour le Myanmar pris d'un seul tenant. La «Birmanie inférieure » a été colonisée progressivement de 1812 à 1852, suivant les deux premières guerres anglo-birmanes. L'espace d'enquête est cependant situé en "Birmanie supérieure », qui fut intégrée à la Birmanie britannique en 1886 au terme de la troisième guerre anglo-birmane.

4 Ce terme renvoie à l'accaparement des terres par des non cultivateurs (Charney, 2009).

5 Le système de quotas fut réduit de deux tiers à la fin des années quatre-vingt et officiellement supprimé en 2003.

6 Comme par exemple pour la délivrance de titres fonciers, l'accès aux projets de développement (routes, électricité, écoles) ou encore l'accès à l'emprunt agricole. Ces pratiques sont comparables à ce qu'ont pu montrer G. Blundo et J.-P. Olivier de Sardan pour le contexte africain (Blundo, 2006). 
ont été votées en 2012 : la Farmland Law pour l'enregistrement de titres de "propriété privée" ", et la Vacant, Fallow, Virgin Lands Management Law qui oriente les conditions d'allocation des terres ainsi qualifiées (Oberndorf, 2012).

Au-delà de ces jalons historiques, il n'y a que très peu d'information disponible sur la mise en œuvre locale de ces politiques. La biographie d'une parcelle permet d'éclairer cette question. Selon Sikor (2008), il faut d'abord déconstruire la catégorie " publique ». Son usage doit être replacé dans une histoire où la diversité des acteurs, des pratiques, des dynamiques historiques, des contextes sociopolitiques, culturels et des conditions agroécologiques donne forme et sens aux relations foncières. Sur le plan juridique, la notion de "public » s'oppose à celle de " privé » dans une dichotomie dominante issue de l'histoire politico-juridique occidentale. Cette définition a largement été introduite durant la colonisation britannique du Myanmar (Furnivall, 1957), pour être ensuite diluée au sein de multiples catégorisations par le gouvernement militaro-socialiste. Dès lors, il est intéressant de confronter la catégorie de « terre publique » avec celles de « terre commune » et " terre villageoise». Dans la littérature foncière, publiciser est l'action de transformer le statut d'un espace ou d'une ressource en transférant sa propriété vers un propriétaire collectif, imaginaire, et souvent incarné par un gouvernement national. À ceci s'ajoute la catégorie de " commun », qui renvoie à la notion de partage de l'accès à une ressource "par un ou plusieurs groupes d'ayants droit, partage sous-tendu par un enjeu d'équilibre entre l'intérêt collectif [...] à assurer le renouvellement de la ressource, et l'intérêt individuel/immédiat de ceux qui partagent » (GRET, 2015, p. 1). À la différence du cas indien ${ }^{8}$ (Chakravarty Kaul, 1990), nous verrons que la terre en question est considérée par les villageois comme une "terre villageoise ", à mi-chemin entre terre commune et terre collective.

La démarche consiste alors à suivre l'histoire d'une terre en se focalisant sur ses changements de statut et d'usage. La reconstitution de la biographie des « choses » comme procédé d'enquête a porté ses fruits depuis les travaux d'Appadurai (1986). L'idée est ici de retracer la vie d'une terre. En effet, le sol est une chose (Hall, 2013) qui, dans sa dimension foncière, peut être analysée comme une propriété ${ }^{9}$ (Benda-Beckmann et al., 2006).

7 L'État reste le propriétaire ultime de toute terre, ce qui continue à freiner l'initiative privée (terres rizicoles dédiées uniquement à la riziculture, cultures saisonnières ne pouvant être transformées en cultures pérennes, etc.).

8 Minoti Chakravarty Kaul analyse les façons dont le régime colonial a impacté l'usage et la régulation de ressources historiquement considérées comme communes.
Après une description détaillée de la biographie du terrain de football du village de Pawn Gyo, le déploiement des pratiques gouvernementales (coloniales, militaro-socialistes, militaristes) est confronté à l'économie morale villageoise à l'œuvre dans les relations foncières de la zone sèche du Myanmar (bouddhisme, arrangements fonciers, répartition des droits, relations de pouvoir). L'accent est ensuite mis sur la situation actuelle pour montrer les enjeux de la définition du statut d'une terre (commune, villageoise, vacante) et la place du chef de village dans les rapports qu'entretiennent les habitants de Pawn Gyo avec le gouvernement.

\section{L'histoire d'une terre agricole devenue terrain de football}

La parcelle se situe à l'extrémité sud du village de Pawn Gyo, lui-même rattaché au groupe de villages de Myin Kyaw Pon ${ }^{10}$. Au début des années cinquante, un couple du village récemment marié demanda à un agriculteur ayant des terres en friche disponibles s'il pouvait en cultiver une partie pour devenir paysans. Suite à la réponse positive de celui-ci, un accord coutumier fut conclu : il s'agit du thon"su'tiq su' ${ }^{\prime 11}$, correspondant à trois parts/une part. La personne qui travaille la parcelle doit fournir un quart de chaque récolte à celle qui détient la terre. Dans ce cas précis, la parcelle a été cultivée selon ces conditions durant une dizaine d'années.

Ces années correspondent à la période de post indépendance (à partir de 1948), marquée par la nationalisation des terres ${ }^{12}$ qui a fait du gouvernement l'unique propriétaire légal de toutes terres. Les individus sont légalement devenus des détenteurs de droits d'usage, délégués (lok-paing-hkwin) par l'État pour cultiver leur parcelle. Cette politique s'est inscrite dans une lutte contre le landlordism visant à allouer les terres à ceux qui les cultivent via la mise en œuvre d'expropriationsréallocations de parcelles. Elle connaît son apogée au

\footnotetext{
9 Le mot «propriété » renvoie ici au concept de «property » utilisé en anglais comme dans «property relations », c'est-à-dire relevant des relations sociales entre acteurs à l'égard des objets / choses de valeur.

10 Les Village-Tracts, ou groupes de villages, ont été créés durant la période britannique selon un plan colonial d'administration directe dont les principaux échelons sont aujourd'hui le Village-Tract (pour l'espace rural), le Ward (pour l'espace urbain), puis le Township (ou commune), et enfin la Division ou le State, ces deux derniers ensembles pouvant être assimilés à des régions, les States renvoyant à des régions ethniques (comme l'État Shan par exemple) (cf. The Village Act, 1907).

11 Transcription conventionnelle avec « accentuation levée » (Okell, 1971).

12 Inspirée par la Constitution de 1947 et mise en acte par le Land Nationalisation Act de 1948, repris en 1953.
} 


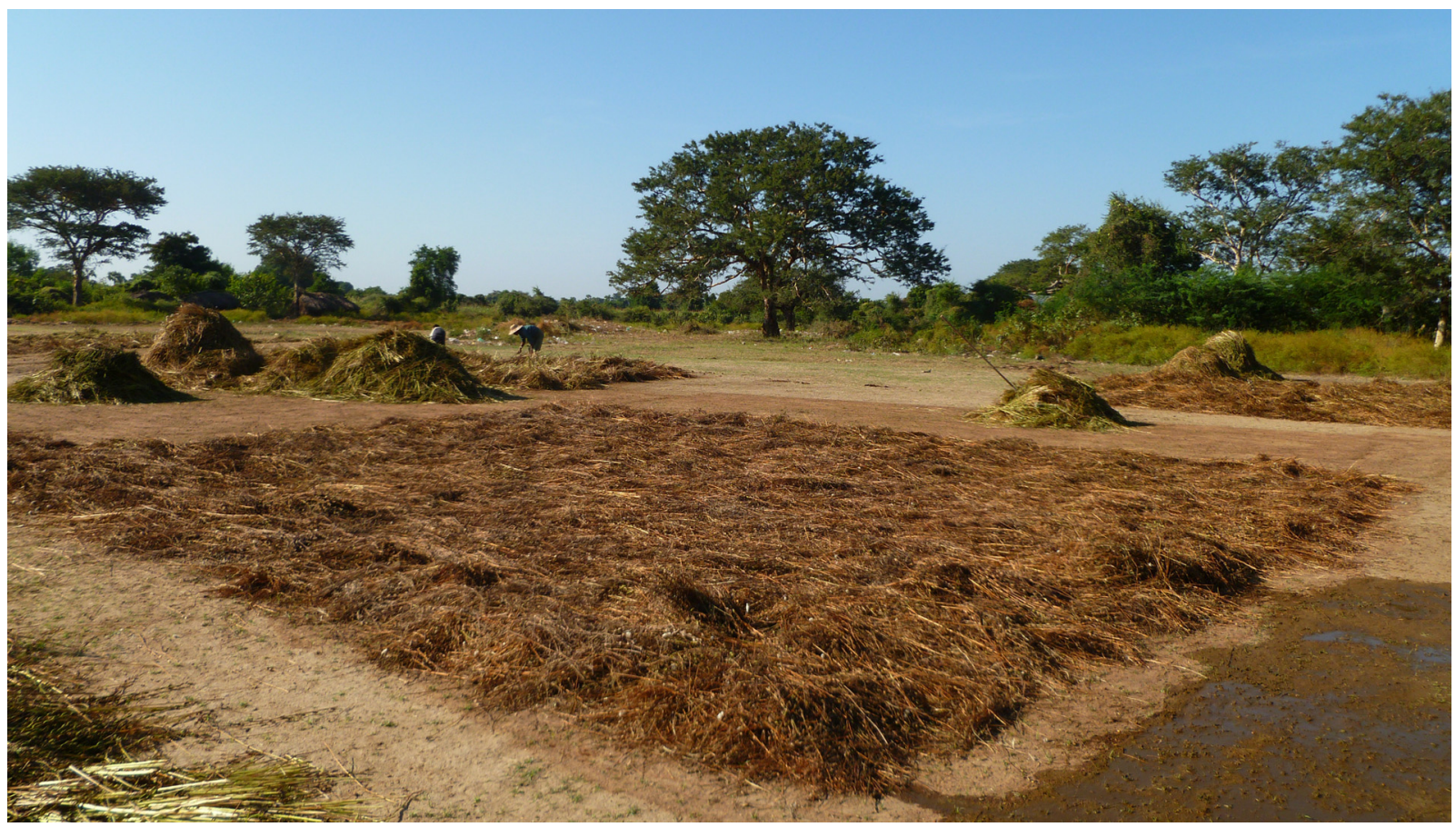

Photo. La parcelle utilisée comme « thalin » sur le terrain de football de Pawn Gyo. (C Stéphen Huard).

niveau local après le coup d'État du général Ne Win en 1962 qui marque le début du gouvernement militarosocialiste.

Dans la présente étude de cas, cette politique a eu pour conséquence le transfert du droit d'usage sur la parcelle des propriétaires initiaux vers le couple. En effet, après cinq années consécutives de mise en culture, ce couple a pu demander de se voir délégué le droit d'usage par le gouvernement. Ce type de transfert ordonné par l'État militaro-socialiste a été mis en œuvre par le Village People Council ${ }^{13}$ présidé par le chef du groupe de villages de l'époque. Le transfert de droits fut ensuite enregistré par le State Land Record Department (SLRD) ${ }^{14}$ de Monywa en charge du cadastrage, du suivi des cultures et de la définition de $1^{\prime}$ usage légal des terres ${ }^{15}$. Cette étape représenta un moment de fortes tensions dans le village, peu de personnes voulant faire état de leurs pertes ou acquis pour cette époque du fait du caractère clientéliste de l'appropriation des terres. Ainsi, l'accord coutumier

\footnotetext{
13 Organe local du gouvernement socialiste présidé par le chef du groupe de villages (vivant à cet endroit).

14 Renommé Department of Agricultural Management and Statistics en 2016.

15 Cette institution a donc le monopole de la définition de l'usage des terres suivant une catégorisation datant de l'époque britannique. Les terres agricoles sont notamment divisées en deux principaux sous-groupes (lei-myei pour les terres irriguées dédiées à la culture du riz, ya-myei pour les terres n'ayant pas accès à l'irrigation).
}

régulant les relations sur cette parcelle a été supplanté par l'action gouvernementale. Une fois la réforme appliquée, le statut des terres et la distribution des droits n'ont pas été contestés plus avant.

Près de vingt ans plus tard, dans les années soixantedix, des agents du gouvernement sont venus au village pour y délimiter différents espaces, notamment pour la construction d'une école publique et d'un terrain de sport. La parcelle en question a été choisie, avec d'autres, pour la création d'un terrain de football. Ainsi, les personnes qui s'étaient vues allouer un droit d'usage sur la terre ont été expropriées. Malgré des tentatives de résistance, elles sont restées impuissantes face aux agents en charge de la régulation foncière (SLRD) et face au chef du groupe de villages en charge du Village People Council pendant la majeure partie de l'époque militaro-socialiste. Ces terres ont été enregistrées officiellement comme terres vacantes ( $p a$ lap myei), c'est-à-dire comme des terres n'ayant pas d'usage agricole, donc à la disposition du gouvernement. La logique de sélection des parcelles à exproprier fut d'ordre spatial - une surface suffisante pour créer un terrain de football - mais aussi d'ordre sociopolitique. Les terres concernées sont en partie celles qui ont subi des expropriations-réallocations. Le chef du village a choisi ces parcelles en raison de l'histoire foncière du village. La transformation du statut de la parcelle a une dimension fondamentalement locale liée à l'histoire du village et constitutive du rapport au gouvernement. 
Une fois le terrain de football délimité, deux phénomènes sont apparus. En hiver, cet espace, non cultivable du fait de son nouveau statut, est utilisé comme thalin", i.e. comme un espace pour battre et faire sécher les récoltes (Photo).Un ré-ancrage des pratiques locales d'usage du sol s'est opéré. D'un côté, les personnes ayant cultivé ces terres avant ce changement gardent un droit d'usage prioritaire sur cet espace - suivant l'ancien tracé de leur parcelle - pour y faire sécher leurs récoltes si elles en ont besoin. De l'autre, les agriculteurs qui ont eu besoin de plus d'espace pour faire sécher leurs récoltes ont utilisé les espaces vacants, s'accaparant alors un droit d'usage prioritaire. Durant le reste de l'année, l'équipe de football du village utilise le terrain. Les anciennes générations de footballeurs faisaient payer sous forme de cagnotte les personnes ayant un thalin" sur le terrain afin de s'acheter des équipements (ballons et maillots). L'équipe actuelle a redonné récemment vie à ce système pour construire des buts en métal. Ainsi, l'équipe de football et les personnes ayant un thalin" sur le terrain de football ont un accord répartissant les droits et leur durée pour l'utilisation de cet espace.

En 2013, le General Administration Department ${ }^{16}$ de Monywa a transmis une lettre au chef du village demandant si lui-même, le comité foncier ${ }^{17}$ et l'ensemble des villageois acceptaient de vendre le terrain de football dans le but de financer l'acheminement de l'électricité au village. Le gouvernement, après avoir transformé cet espace en terre vacante, mettait en avant ce statut juridique pour permettre la vente. Après consultation des chefs de dix maisonnées et des villageois réunis, l'opinion du village était favorable au projet : vendre le terrain de football et ainsi récupérer les 80000000 kyats $^{18}$ nécessaires pour réaliser les travaux. Le chef du village est ensuite retourné voir son supérieur direct. Ce dernier lui a conseillé de ne pas vendre le terrain de football et d'attendre les prochains programmes de développement. Pour celui-ci, la décision de vendre et les problèmes qui pourraient en surgir seraient de la seule responsabilité du chef de village, malgré un accord écrit signé par chaque chef de maisonnée si la vente était approuvée. Dès lors, le chef du village a demandé au SLRD d'attribuer officiellement cette terre au village dans un contexte de forte augmentation du prix des terres. Cet épisode est décisif pour comprendre l'imbrication des différentes conceptions quant aux statuts et usages d'une terre. Après plusieurs étapes (réallocation, expropriation, transformation en terre vacante), cet

16 Colonne vertébrale de l'administration gouvernementale depuis les années quatre-vingt.

17 Officiellement appelé le Village Land Management Committee (VLMC), cet organe a été créé localement pour réguler les potentiels problèmes survenant lors de la réforme foncière de 2012.

18 Environ 75000 dollars US. espace est à la fois considéré comme terrain de foot (bollon kwet), comme espace pour sécher les récoltes (thalin"), comme terre commune (ywa bon" myei) et comme terre villageoise (ywa paing myei).

\section{Pratiques gouvernementales vs relations sociales locales: les deux faces d'un même contexte}

Le sol, dans sa dimension foncière, est enchâssé dans des relations de propriété qui sont composées d'une large variété d'arrangements en termes de droits et d'obligations à travers le temps. Ces arrangements peuvent être conceptualisés comme "faisceaux de droits » (LavigneDelville, 2010). Selon Benda-Beckmann et al. (2006), ce sont les interactions entre pratiques sociales «[which] create, maintain and change what property is, having differenciated effects at the level of ideologies, of legally institutionalised categorical property relations, and of concretised property relations » (p. 15). L'ensemble de ces arrangements s'exprime dans les idéologies et idéaux culturels, au sein des institutions politico-juridiques et dans les relations et pratiques sociales.

Concernant le terrain de football de Pawn Gyo, il est alors intéressant de confronter les façons dont ont été légitimées et appliquées les politiques du gouvernement avec l'ancrage des relations foncières locales. Ces deux ensembles ne sont pas isolés l'un de l'autre. Ils sont en constante interaction et la position du chef de village concernant la vente de cette parcelle révèle cette imbrication.

\section{Déploiement local des pratiques gouvernementales}

Depuis la colonisation britannique, à partir de 1886 pour notre zone d'enquête placée sous administration directe (Furnivall, 1957), les gouvernements successifs ont encadré les relations foncières par différents moyens ${ }^{19}$. Le statut « commun » ou « villageois » d'une terre pose alors la question du rapport à l'État, dans la mesure où les gouvernements birmans après l'indépendance ont repris les outils de la régulation foncière coloniale pour développer leurs politiques (Huard, 2014). Le régime colonial a imposé un système administratif et judiciaire reposant sur le Rule of Law (Cheesman, 2010).

\footnotetext{
${ }^{19}$ Ces moyens sont l'établissement d'un cadastrage imposant de nouveaux statuts aux terres, la définition des transferts légaux, la mise en place et l'abandon des cours de justice, l'imposition de chefs de village sur des espaces de juridiction décontextualisés, la création de comités pour l'application des politiques agraires, la mise en œuvre d'un réseau de coopératives, etc.
} 
La diversité des statuts fonciers a été transcrite et simplifiée en deux catégories - terres étatiques et terres non étatiques - afin de développer une régulation foncière basée sur une administration déconcentrée ${ }^{20}$. C'est à cette époque que la catégorie "publique » est apparue au Myanmar, renvoyant aux propriétés détenues par l'État. Plus concrètement, la colonisation a créé un système de reconnaissance de propriété, de cadastrage et de taxation basé sur une division du territoire en juridictions (ici le groupe de villages) et une unité minimale de propriété privée ( $u$ paing) le plus souvent accordée au chef de maisonnée. Pour notre étude de cas, le propriétaire initial disposait d'un droit de propriété reconnu via l'enregistrement de son nom avec le numéro de parcelle dans les registres du SLRD. La catégorie " public property » a ensuite été créée pour qualifier tout ce qui est matériellement utilisé pour le fonctionnement du gouvernement ${ }^{21}$.

À l'indépendance, l'héritage juridique britannique a été couplé à une relecture des sources du droit birman : le Dhammathat (un ensemble de règles ayant pour objets la société et l'univers) et le Yazathat (la loi du roi). Ces deux textes définissent le sol, l'espace terrestre, comme une propriété universelle. Sa gestion doit prendre en compte ce côté universaliste pour éviter un déséquilibre sociétal. La nationalisation des terres s'est alors appuyée sur une structure étatique moderne associée à cet ensemble juridique. Cela a légitimé l'appropriation de l'espace national par le gouvernement, seul représentant de la nation. Parallèlement, ce régime supprima officiellement les intermédiaires non gouvernementaux entre l'État et les agriculteurs. De la production à l'exportation, en passant par l'approvisionnement, l'ensemble des relations économiques et sociales autour de l'agriculture ont été pensées dans une relation directe entre l'État et les populations paysannes ${ }^{22}$. Comme toute terre était officiellement étatique, la catégorie " terre publique » a été diluée au sein de multiples classifications visant à qualifier et contrôler l'usage des terres (Leckie et Simperingham, 2009). Le statut légal de "terre villageoise » ne semble pas avoir été changé depuis sa définition coloniale : " "village" means an area appropriated to dwelling places not included in the limits of a town " (The Lower Burma Town and Village Lands Act, 1898).

Après le coup d'État de 1962, le gouvernement militaro-socialiste a fixé de nouvelles conditions de transfert des droits fonciers et a continué la politique

\footnotetext{
${ }^{20}$ Cf. The Village Act, 1907 et The Upper Burma Land and Revenue Regulation, 1889.

21 Cf. The Public Property Protection Act, 1947.

22 Théorie notamment développée par deux textes : « La voie birmane vers le socialisme » (1962) et «La philosophie du parti du programme socialiste birman: le système de corrélation entre l'homme et son environnement » (1963).
}

d'expropriations-réallocations ${ }^{23}$ de terres. Il a rendu illégaux les arrangements entre tenants (locataires) et landlord (propriétaire) (Charney, 2009). Dans cette lutte contre le landlordism, le comité foncier villageois, avec le chef de village à sa tête, est devenu le premier niveau de régulation de l'usage des terres. Les personnes qui étaient tenants pouvaient alors se voir attribuer un droit d'usage délégué sur la terre qu'ils cultivaient en continu pendant cinq ans ${ }^{24}$. C'est cela même qui s'est passé pour notre couple. Le comité foncier villageois a appliqué cette politique de façon ad hoc, i.e. en fonction des relations foncières et des rapports de pouvoir dans le village de Panw Gyo. Certaines personnes considérées comme grands propriétaires (myei shin) ont dû céder leurs terres cultivées en faire-valoir indirect ${ }^{25}$, selon l'arrangement thon"su'tiq su' (trois parts/une part) dans le cas étudié ici.

Lorsque le gouvernement est intervenu dans les années soixante-dix pour changer le statut de certains espaces (école, terrain de football, etc.), il s'est à nouveau appuyé sur les institutions locales. Le Village People Council, présidé par le chef du groupe de villages de l'époque, a dû choisir quelles terres seraient confisquées. D'un côté, le fait que les terres réquisitionnées pour la création du terrain de football soient en partie celles qui ont été impactées par la politique de lutte contre le landlordism ne relève pas du hasard. Une hypothèse serait que le nouveau chef du village ait voulu « inverser » la politique d'allocation des terres aux tenants en expropriant ceux qui auparavant ont pris les terres de grands propriétaires. Cela renvoie aux relations villageoises axées sur la distinction entre taung-thu (agriculteur disposant de ses propres moyens de production) et myaukthu (villageois ne possédant pas de terre agricole). Le couple de villageois a donc été exproprié afin de créer un terrain de football. D'un autre côté, ce terrain de football a été classifié en terre vacante (pat lap myei). Officiellement, ce statut renvoie aux terres n'ayant pas encore ou plus de vocation agricole (Oberndorf, 2012). Il semble que, localement, il fut attribué à la discrétion de l'agent du SLRD. L'utilisation de cette catégorie juridique est à replacer au sein de l'économie politique nationale orientée vers l'agriculture avec un État possesseur de toute terre, qui les classe en fonction de leur usage agricole. Ayant permis de nombreuses confiscations à l'échelle du pays (Hudson-Rodd et al., 2008 ; Woods, 2011), la classification en terre vacante montre dans le cas présent l'absence de reconnaissance juridique d'espaces communs ou villageois.

\footnotetext{
23 Politique orientée par les lois et règlements fonciers de 1963.

24 Instruction gouvernementale 1/64 datant de 1964.

25 Voir Colin (2004) pour une présentation synthétique des formes de transfert de droits.
} 


\section{Relations foncières et économie morale villageoises}

Tout d'abord, le bouddhisme structure la conception de l'espace villageois et son agencement topographique. Le monastère au nord et le cimetière au sud-est façonnent l'espace villageois. L'est, direction vers laquelle sont orientées les représentations du bouddha, est pensé comme une entrée pour les influences positives, une « entrée de la chance » (min-gala-pauk) matérialisée par des chemins selon le plan des anciens qui ont bâti le village. Respecter cette ouverture revient à respecter les choix des ancêtres pour " pousser, améliorer » la chance du village. Le référent bouddhiste participe de la conceptualisation de ce qui est dans et hors du village. Cependant, le terrain de football est considéré par certains comme ne faisant pas partie du village, car il ne date pas de sa création, et par d'autres comme une partie intégrante car il appartient à tous (amya' paing-myei).

À côté de ces conceptions, divers arrangements concernant l'usage de cette terre se sont succédés. Le type d'arrangement existant avant l'intervention du gouvernement dans les années soixante, de la forme thon"su'tiq $s u^{\prime}$, révèle l'ancrage agroécologique et sociopolitique de l'économie morale paysanne de la zone sèche du Myanmar. Cet accord est largement répandu dans cette région depuis au moins le $19^{\mathrm{e}}$ siècle (Furnivall, 1957). Sa régulation s'appuie sur l'ancrage des normes et pratiques coutumières qui sous-tendent les relations foncières locales (Huard, 2014). La clef de répartition de l'accord - division des récoltes en quatre portions, dont une « rendue » au maître de la terre - est perçue comme juste car elle assure au propriétaire une part minimum malgré les aléas climatiques (variabilité des pluies et fortes chaleurs) qui empêchent de prédire les récoltes futures.

Cette répartition en portions structure divers arrangements concernant les transferts temporaires de droits fonciers. Elle permet à un propriétaire de mettre une terre en valeur sans avoir à - ou sans pouvoir - la cultiver. $C^{\prime}$ est une forme de faire-valoir indirect où les droits d'accès et d'usages sont transférés de la personne qui détient l'autorité sur la terre vers celle qui va cultiver la parcelle ${ }^{26}$. Cette pratique repose sur des droits et obligations réciproques ${ }^{27}$ ainsi que sur certaines conceptions de ce que signifie gérer une terre (ya-myei-htein"thein"yan), ou encore en être maître (ya paing-shin). En outre, depuis au moins l'époque précoloniale, pour devenir tenant, la personne qui gère la terre s'inscrit dans une longue relation avec celui qui en est maître, relation pouvant durer plusieurs années avant que l'accord devienne pérenne.

\footnotetext{
26 Généralement le mari s'il s'agit d'un couple, mais il peut s'agir d'une femme si elle est seule ou veuve.

27 Pour une analyse fine de ce que recouvrent ces catégories, voir Huard (2014).
}

Le propriétaire ne confiera pas sa terre à une autre personne pour la mettre en valeur si le locataire actuel assure chaque année un travail de qualité et de bonnes récoltes. Ou encore, le propriétaire ne disposant pas des moyens de mettre en culture toutes ses terres recevra de son tenant une partie de la production. L'arrangement thon"su'tiq su' permet d'agencer les termes de l'accord, ceux-ci variant en fonction des rapports de pouvoir et de la situation socioéconomique des contractants.

Dans la zone sèche du Myanmar, la lutte contre le landlordism à partir des années soixante a permis dans une certaine mesure l'appropriation de terres par les personnes considérées légalement comme tenants. Localement, en donnant la possibilité de demander le transfert du droit d'usage sur une parcelle cultivée pendant au moins cinq ans, cette politique s'est confrontée aux relations foncières villageoises, notamment axées sur la distinction entre taung-thu et myauk-thu. En effet, l'histoire de l'implantation du village, de sa distribution foncière, des relations interfamiliales et matrimoniales met en avant l'importance des relations de dépendance, d'exclusion et d'entraide entre les familles et maisonnées en partie autour de la dichotomie taung-thu et myauk-thu ${ }^{28}$. Cette catégorisation met l'accent sur la possession des moyens de mettre en valeur les terres agricoles. La possession de bétail (et l'approvisionnement en fourrage), d'outillage, d'espace de stockage, etc., fait d'une personne un «vrai » agriculteur (taung-thu). Un agriculteur est en mesure de mettre à profit ses moyens de production ${ }^{29}$, d'accumuler des terres, de l'or, de capter la main-d'œuvre, et de créer des relations de dépendance à travers l'usage de ses moyens de production et sa capacité à financer (via des prêts, des hypothèques, des donations) les activités agricoles et devoirs religieux $\mathrm{d}$ 'autres villageois (noviciat bouddhique pour les jeunes garçons par exemple) ${ }^{30}$. L'allocation des terres aux tenants s'est confrontée à cet ensemble de relations villageoises ancrées dans une temporalité longue, à tout le moins différente de celle des politiques et réformes successives.

Ainsi, avant d'être qualifiée de "vacante », la parcelle en question dans cette étude de cas était dépendante de relations foncières spécifiques comprenant une

\footnotetext{
28 Cette différence de statut entre taung-thu et myauk-thu comme mode d'organisation social villageois est actuellement affectée par la multiplication des possibilités de travail dans et hors des villages, les migrations annuelles et saisonnières, l'augmentation exponentielle du prix des terres, l'accroissement démographique, la disparition des terres vacantes et les transformations des conceptions quant à la transmission du patrimoine.

29 Par exemple en louant ses outils ou son bétail.

30 Pour d'autres exemples de cette structuration des rapports sociaux, voir Boutry (2015).
} 


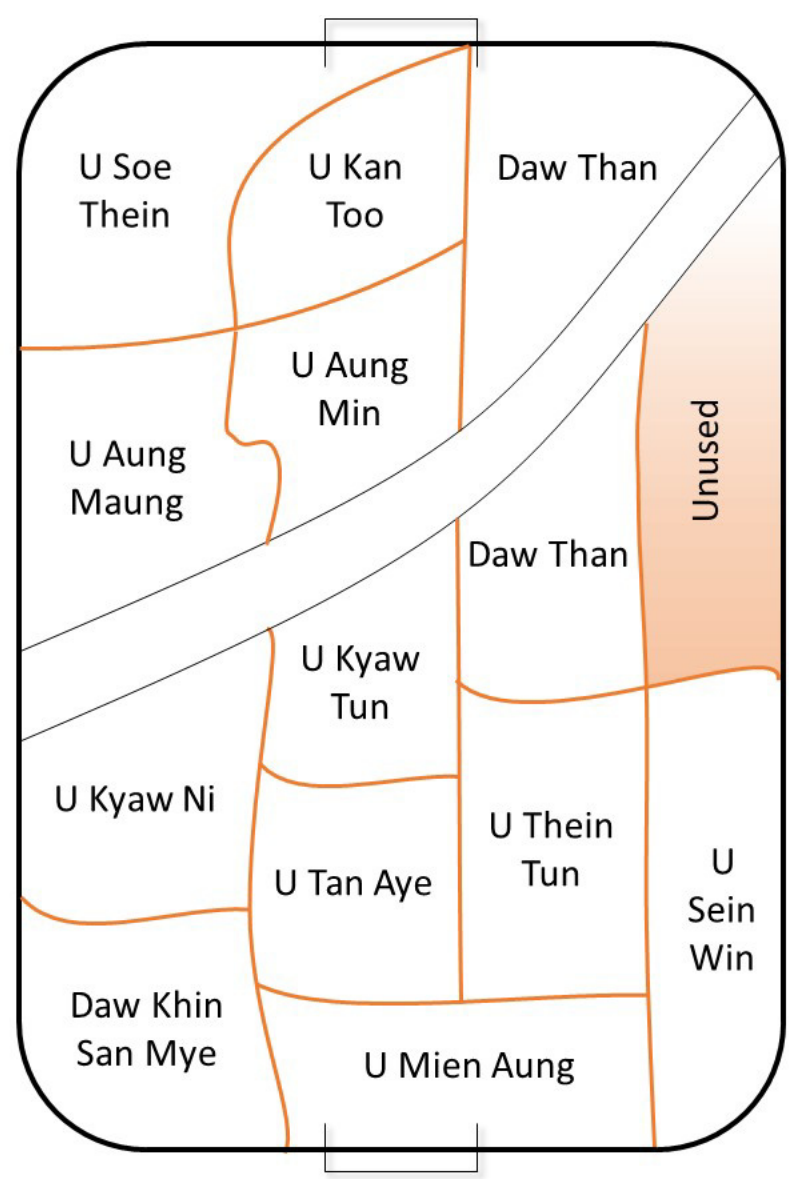

Figure. Répartition des agriculteurs utilisant le terrain de football pour leurs récoltes. (Source : Stéphen Huard)

dimension d'entraide, d'intégration dans le village, de dépendance, d'exclusion foncière ou de clientélisme par exemple. Après sa transformation en terrain de football, on assiste rapidement à un réancrage des pratiques locales. Les personnes qui ont été expropriées disposent $\mathrm{d}^{\prime}$ un droit d'accès prioritaire afin d'utiliser cette terre comme thalin" (espace de séchage), ainsi que la capacité de déléguer cet usage. Cet ensemble de droits est étendu au patrimoine de la famille, couple ou personne qui l'utilise. À ce titre, il est intégré aux dynamiques de transmission de l'héritage (amue) : les parents doivent en théorie transmettre égalitairement l'ensemble de leur patrimoine à leurs enfants au moment de leur mariage ou avant de décéder (Huard, 2014) ${ }^{31}$. La dimension intergénérationnelle de la transmission, les conceptions autour de ce qui est juste et légitime ${ }^{32}$ et les contraintes pour assurer la subsistance des familles structurent les arrangements et les stratégies possibles pour garantir la

\footnotetext{
31 Voir également le rapport d'étude du GRET qui sera publié en 2017 et développé par Boutry M., Allaverdian C., Mellac M., San Thein et Tin Myo Win intitulé Land tenure in rural lowland Myanmar. Understanding rural land issues to engage comprehensive policy dialogue in Myanmar.
}

continuité d'un patrimoine, ou bien pour capter celui d'une autre famille (à travers le mariage ou l'adoption par exemple). Ainsi, le terrain de football, constitué notamment de la parcelle allouée au couple de tenants, est composé de multiple thalin" appartenant à différentes familles de grands agriculteurs et transmis généralement vers l'enfant qui prendra en charge ses parents et leurs terres (Figure).

À cette répartition des espaces de séchage entre agriculteurs sur cette parcelle s'ajoute son utilisation par l'équipe de football. Dans le centre du Myanmar, chaque village voit ses jeunes organiser des tournois locaux. Un des moyens pour l'équipe du village de financer l'achat de matériel (maillots, ballons) est de taxer (en nature après les récoltes) les personnes utilisant le terrain pour leurs récoltes. Cette pratique est tombée en désuétude entre les deux dernières générations et a été remise au goût du jour récemment pour construire des buts en métal. Les jeunes footballeurs sont allés de maisonnée en

\footnotetext{
32 Comme l'égalitarisme de la répartition, le fait que la personne prenant en charge les parents récupère l'espace d'habita-
} tion, etc. 
maisonnée pour collecter cette taxe (approuvée au préalable par les personnes les plus influentes du village). Ainsi, l'équipe de football villageoise dispose d'un droit $d^{\prime}$ usage du terrain en parallèle de son utilisation par certains agriculteurs. La conception locale du juste est à l'œuvre dans cet arrangement : il est normal que les personnes utilisant le terrain de football participent à son financement. Ainsi, à l'échelle villageoise, cet espace est aujourd'hui utilisé de plusieurs façons (football, séchage des récoltes) qui renvoient aux manières dont est géré un bien villageois (certaines personnes influentes accordent et/ou définissent les usages légitimes en fonction des coutumes et enjeux locaux).

Parallèlement, cet espace est à la fois considéré comme une terre commune (ywa bon"myei) et une terre villageoise (ywa paing myei). À la différence de son statut étatique qui ignore les réalités locales, la manière de concevoir cet espace et ses usages est fluide et ancrée dans les relations villageoises. Son statut reste cependant négociable, notamment lorsque les projets du gouvernement entrent en scène.

\section{Terre villageoise ou terre vacante? Quand l'ambiguïté autour du statut d'une terre est aux prises avec les relations politiques locales}

En 2013, le General Administration Department de Monywa a proposé au village de vendre le terrain de football pour financer l'acheminement de l'électricité. Le chef du village avait alors consulté les villageois, dont l'opinion était favorable au projet. Ce projet est cependant resté sans suite : la décision de vendre et les problèmes qui pourraient en surgir relevant de la seule responsabilité du chef de village, celui-ci, malgré un accord signé par chaque tête de maisonnée approuvant l'accord général de vente, a refusé de donner suite au projet.

Plusieurs raisons peuvent expliquer ce refus. L'acheminement de l'électricité pourrait être organisé plus tard et financé par des programmes régionaux de développement. Dès lors, la vente du terrain de football est une solution à court terme qui n'est pas sans risque. Par exemple, le chef de village de 2013, s'il n'était pas renouvelé en 2016 (ce qui fut le cas), devrait assumer ses décisions antérieures sans la protection de la fonction. Quand un villageois devient chef de village, il noue de bonnes relations avec les villageois s'il signe pour eux leurs demandes, s'il prend le temps de regarder la validité des revendications par exemple. Quand il redevient un villageois «lambda ", les animosités peuvent ressurgir : il pourrait être accusé d'avoir falsifié l'accord, d'avoir forcé la vente ou de l'avoir orientée à son profit en cas de conflit interne au village, dans un contexte d'augmentation récente du prix des terres. Étant issu d'une famille aisée, il peut craindre que certaines personnes viennent lui « manger son argent » en entamant des procédures juridiques. De plus, l'histoire de cette terre, ses usages successifs et concomitants, offrent un terreau propice aux revendications : les personnes expropriées, les anciens tenants, l'équipe de football et les agriculteurs utilisant cet espace pour leurs récoltes ont chacun à leur façon la possibilité de contester la vente (comme arguer d'un usage collectif ou faire valoir un droit de propriété). Dès lors, l'éventualité d'un conflit met en avant la position du chef du village : il est à la fois le catalyseur des projets du gouvernement au niveau local - et en retire des avantages matériels et sociaux - mais sa responsabilité est seule engagée dans leur mise en œuvre.

Cet épisode met en avant les contradictions entre le statut légal de la terre - vacante - et ses statuts locaux commune, villageoise. Ces contradictions sont en partie le fruit des politiques et projets du gouvernement pour réguler l'usage des terres. Après avoir abandonné sa politique socialiste au profit de l'agrobusiness, le gouvernement a délaissé les terres villageoises jusqu'au moment où un enjeu de développement est venu actualiser la question du statut local des terres. Ainsi, le statut du terrain de football est enchâssé dans les relations sociopolitiques locales (régulation d'un bien villageois) et les pratiques gouvernementales (contrôle des terres, clientélisme). La possibilité de vendre cette terre renvoie à la façon dont son statut est négocié. C'est parce que le terrain de football est majoritairement défini comme terre villageoise que l'avis du village a été pris en compte. $C^{\prime}$ est à cause des conflits potentiels engageant la responsabilité du chef du village que la vente a avorté.

Le statut de cette terre est donc ambigu. Il se construit au gré des événements. Au sens de l'anthropologie du foncier, le statut de cette terre navigue entre terre collective et terre commune : son appropriation collective (terre possédée par tous les villageois) est revendiquée face à certains enjeux, mais la majorité du temps ce sont surtout deux groupes qui en partagent l'accès et participent à son entretien (caractère de partage et de reproduction). Cette ambiguïté théorique reflète les risques possibles en cas de vente pour le chef de village : 1'appropriation collective est revendiquée par tous a priori mais l'histoire de la terre, son usage commun au profit de deux groupes et l'augmentation des prix offrent un terrain propice aux revendications.

\section{Une histoire par à-coups, un statut en millefeuille}

$\mathrm{Au}$ fil de cette recherche, les différentes dimensions dans lesquelles le terrain de football de Pawn Gyo est (a 
été) intégré sont apparues. D'un côté, l'histoire de cette parcelle est un exemple de la façon dont le foncier local est réaménagé par les pratiques gouvernementales. $\mathrm{Du}$ cadastrage des terres pour leur taxation à l'époque britannique à la redistribution des terres aux tenants par un ancien chef de village, en passant par leur nationalisation, ce sont autant de politiques gouvernementales qui ont tenté de cadrer les usages du terrain de football de Pawn Gyo. De l'autre, la biographie de cette terre révèle en partie l'économie morale villageoise. Le référent bouddhique dans les représentations de ce qui est dans ou hors du village, les multiples arrangements fonciers reflétant aussi bien les relations sociopolitiques, les conceptions de la propriété et sa transmission, des transferts de droits, du juste et du légitime, les rapports de dépendance, d'exclusion et d'entraide ou encore la question de la régulation villageoise de l'accès à un bien sont autant de dimensions à considérer pour comprendre la situation actuelle.

L'usage de la force, l'imposition de nouveaux droits et statuts sur les terres ainsi que les restrictions quant à l'usage légal des ressources sont des éléments nécessaires à la compréhension de l'histoire locale. Cependant, les relations foncières sont façonnées par les normes et pratiques locales qui composent avec les pratiques gouvernementales. Un parallèle est possible avec les analyses de James Scott qui, considérant les villageois comme des acteurs capables de résistance (1985), et l'État en tant que producteur de catégorisations et acteur du changement social (1998), finit par analyser les interactions entre les relations sociales locales et les projets gouvernementaux comme ce qui donne forme à des dynamiques sociétales (2009).

Ainsi, l'absence de reconnaissance officielle des pratiques locales fait que les enjeux de développement actualisent la question du statut d'un espace et font apparaître les forces à l'œuvre pour l'accès ${ }^{33}$ à la terre. Les politiques gouvernementales offrent la possibilité aux acteurs locaux de réaffirmer ou de contester une situation en fonction de leurs intérêts (Lund, 2009 ; Sikor et Lund, 2009). L'intérêt d'acheminer l'électricité par la vente du terrain de football semble être supérieur aux intérêts de l'équipe de football et des agriculteurs l'utilisant pour leurs récoltes. En réaction, le village a revendiqué le statut de terre villageoise appartenant à tous : la capacité des villageois à décider l'aliénation d'une parcelle entérine et crée dans le même temps son statut d'espace collectif. Néanmoins, la vente sera avortée du fait des tensions possibles entre le clientélisme foncier et les risques (et opportunités) associés à la position de chef de village.

Dès lors, les usages et les statuts actuellement accordés à cette parcelle (terrain de football, espace de

\footnotetext{
33 Accès est ici entendu comme la capacité à bénéficier des ressources (Ribot et Peluso, 2003).
}

séchage, terre villageoise) confortent les intérêts de différents groupes (équipe de football, grands agriculteurs, village) et répondent à plusieurs enjeux (faire vivre le football villageois, faire sécher les récoltes à temps, acheminer l'électricité). L'héritage des changements d'usages et de statuts de cette terre, leurs inscriptions dans l'économie morale du village et les modes actuels de gouvernance foncière participent de cette multiplicité de statuts possibles et négociables.

\section{Remerciements}

Merci à mes amis birmans pour leur patience et leurs réponses. Je remercie aussi Pierre-Yves Le Meur, Maxime Boutry, et Léa Vollet pour leurs commentaires sur ce texte. Merci enfin aux relecteurs pour leurs précieux conseils.

\section{Références}

Appadurai A. (Ed.), 1986. The social life of things: commodities in cultural perspective, Cambridge, Cambridge University Press.

Benda-Beckmann F., Benda-Beckmann K., Wiber M.G. (Eds), 2006. Changing properties of property, New York, Berghahn Books.

Blundo G., Olivier de Sardan J.-P. (Eds), 2006. Everyday corruption and the state: citizens and public officials in Africa, London, Zed Books.

Boutry M., 2015. Trajectoires littorales de l'hégémonie birmane (Irrawaddy, Tenasserim, Sud Thaïlande), Bangkok, Irasec-Les Indes savantes.

Chakravarty Kaul M., 1990. Common lands in colonial Punjab: continuity and change. Communication paper, Workshop in Political Theory and Policy Analysis Colloquium, 15 October, Bloomington (IN).

Charney M.W., 2009. A history of modern Burma, Cambridge, Cambridge University Press.

Cheesman N., 2010. Thin rule of law or un-rule of law in Myanmar? Pacific Affairs, 82, 4, 597-613.

Colin J.-P., 2004. Droits fonciers et dimension intrafamiliale de la gestion foncière, Document de travail de l'Unité de Recherche 095, IRD-RÉFO, 8.

Fassin D., 2009. Les économies morales revisitées, Annales. Histoire, sciences sociales, 6/2009, 1237-1266.

Furnivall J.S., 1957. An introduction to the political economy of Burma, Rangoon, Burma Book Club.

GRET (Groupe de recherches et d'échanges technologiques), 2015. Vers la construction d'un cadre analytique et opérationnel sur les communs, Comité technique Foncier $\mathcal{E}$ développement, Les notes de synthèse, 19, 1-9, http:// www.foncier-developpement.fr/wp-content/uploads / Note-de-synthese-19-2.pdf.

Hall D., 2013. Land, Cambridge, Polity Press. 
Huard S., 2014. La régulation locale des conflits fonciers (Birmanie centrale). Mémoire de Master, Marseille, EHESS.

Hudson-Rodd N., Nyunt, M., Thamain T., Htay S., 2008. The impact of confiscation of land, labor, capital assets and forced relocation Burma by the military regime, http:// www.burmalibrary.org/docs/land_confiscation1-20.pdf.

Kurosaki T., Ikuko O., Kyosuke K., Koichi F., 2004. Rich periphery, poor center: Myanmar's rural economy under partial transition to a market economy. Technical report, Tokyo, Hitotsubashi University/COE program, https://hermesir.lib.hit-u.ac.jp/rs/bitstream/10086/14041/1/D0323.pdf.

Lavigne-Delville P., 2010. Conceptions des droits fonciers, récits de politiques publiques et controverses. Les Plans fonciers ruraux en Afrique de l'Ouest, in Colin J.-P., Le Meur P.-Y. et Léonard E. (Eds), Les politiques d'enregistrement des droits fonciers : du cadre légal aux pratiques locales, Paris, Karthala, 64-109.

Le Meur P.Y., 2002. Approche qualitative de la question foncière, Note méthodologique, Document de travail de l'Unité de Recherche 095, IRD-RÉFO, 4.

Leckie S., Simperingham, E., 2009. Housing, land, and property rights in Burma: The current legal framework, Geneva, HLP Institute.

Lund C., 2009. Recategorizing "public" and "private" property in Ghana, Development and Change, 40, 1, 131-148.

Oberndorf R.B., 2012. Legal review of recently enacted farmland law and vacant, fallow and virgin lands management law, Improving the legal $\mathcal{E}$ policy frameworks relating to land management in Myanmar, Food Security Working Group (FSWG) Land Core Group, http://www.foresttrends.org/documents/files/doc_3274.pdf.

Okell J., 1971. A guide to the romanization of Burmese, London, Royal Asiatic Society.

Reçu le 12 octobre 2015. Accepté le 6 octobre 2016.
Ribot J.C., Peluso N.L., 2003. A theory of access, Rural Sociology, 68, 2, 153-181.

Scott J.C., 1977. The moral economy of the peasant: rebellion and subsistence in Southeast Asia, Yale, Yale University Press.

Scott J.C., 1985. Weapons of the weak: everyday forms of peasant resistance, Yale, Yale University Press.

Scott J.C., 1998. Seeing like a state: how certain schemes to improve the human condition have failed, Yale, Yale University Press.

Scott J.C., 2009. The art of not being governed: an anarchist history of upland Southeast Asia, Yale, Yale University Press.

Sikor T., 2008. Public and private in natural resource governance: a false dichotomy?, London, Routledge.

Sikor T., Lund C., 2009. Access and property: a question of power and authority, Development and Change, 40, 1, 1-22.

Steinberg D.I., 1981. Burma's road toward development: growth and ideology under military rule, Colorado, Westview Press.

Taylor R.H., 2009. The State in Myanmar, Honolulu, University of Hawaii Press.

Thant Myint-U, 2001. The making of modern Burma, Cambridge, Cambridge University Press.

Thompson E. P., 1971. The moral economy of the British crowd in the eighteen century, Past and Present, 50, 76-136.

Warr P.G., 2000. The failure of Myanmar's agricultural policies, Southeast Asian Affairs, 219-238.

Woods K., 2011. Ceasefire capitalism: military-private partnerships, resource concessions and military-state building in the Burma-China borderlands, Journal of Peasant Studies, 38, 4, 747-770.

Woods K., 2013. The politics of the emerging agro-industrial complex in Asia's 'final frontier': the war on food sovereignty in Burma, conference paper in Food sovereignty: a critical dialogue, Internal conference Yale University, 14-15 September, Yale.

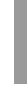

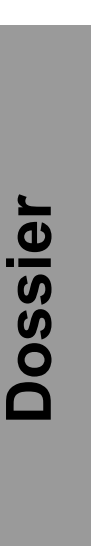

